\title{
Flow of a micropolar fluid bounded by a stretching sheet
}

\author{
A. Desseaux* N. A. Kelson ${ }^{\dagger}$
}

(Received 7 August 2000)

\begin{abstract}
We consider boundary layer flow of a micropolar fluid driven by a porous stretching sheet. A similarity solution is defined, and numerical solutions using Runge-Kutta and quasilinearisation schemes are obtained. A perturbation analysis is also used to derive analytic

* Institut Universitaire de Technologie, Valenciennes, FRANCE. mailto: Andre.Desseaux@univ-valenciennes.fr

$\dagger$ Centre in Statistical Science and Industrial Mathematics, Queensland University of Technology, Australia. mailto:n.kelson@fsc.qut.edu.au

${ }^{0}$ See http: //anziamj . austms .org. au/V42/CTAC99/Dess for this article and ancillary services, (c) Austral. Mathematical Soc. 2000. Published 27 Nov 2000.
\end{abstract}


solutions to first order in the perturbing parameter. The resulting closed form solutions involve relatively complex expressions, and the analysis is made more tractable by a combination of offline and online work using a computational algebra system (CAS).

For this combined numerical and analytic approach, the perturbation analysis yields a number of benefits with regard to the numerical work. The existence of a closed form solution helps to discriminate between acceptable and spurious numerical solutions. Also, the expressions obtained from the perturbation work can provide an accurate description of the solution for ranges of parameters where the numerical approaches considered here prove computationally more difficult.

\section{Contents}

1 Introduction

C538

2 Defining Equations

C539

3 Perturbation Analysis

C543

3.1 Zeroth Order Solution $(n=0)$ for $F_{1}$ and $F_{2} \ldots \ldots$ C544

3.2 First Order Solution $(n=1)$ for $F_{1} \ldots \ldots$. . . . . C546

3.2.1 Complementary Function . . . . . . . . . C546

3.2.2 Particular integral . . . . . . . . . . C548

4 Numerical method

C551 
5 Results

C553

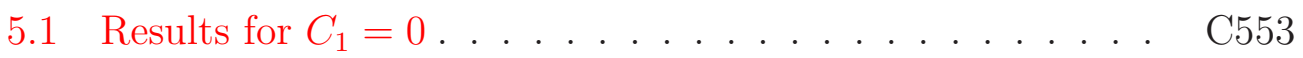

5.2 Results for $C_{1} \neq 0 \ldots \ldots \ldots \ldots \ldots$. . . . . . . . . . . . . . . . . . . . .

6 Conclusion

C558

References

C559

\section{Introduction}

Micropolar fluids are those which contain micro-constituents which can undergo rotation, the presence of which can affect the hydrodynamics of the flow so that it can be distinctly non-Newtonian. The theory of this class of fluids was originally formulated by Eringen [3] in the context of continuum fluid dynamics. Eringen's theory has provided a good model to study a number of complicated fluids, including the flow of low concentration suspensions, liquid crystals, blood, and turbulent shear flows. However, for real, non-trivial flows the theory must be formulated in terms of a system of 19 partial differential equations in 19 unknowns [1]. This has lead subsequent researchers to consider subclasses of microfluids and/or simplified flow situations which are more easily amenable to solution.

In this work, the steady isothermal flow of a micropolar fluid driven by a continuous porous surface is analysed via perturbation and numerical meth- 
ods. The two-dimensional boundary layer flow caused by a moving plate or a stretching sheet is of interest in manufacture of sheeting material through an extrusion process. Tape casting, for example, is an important forming operation commonly used to prepare multilayer capacitors and packages in the ceramic industry. In this process a well-mixed ceramic slurry is usually placed in a container with a rectangular outlet made of parallel walls and the tape casting head is set in motion on a flat substrate. A theoretical treatment from the viewpoint of designing the process and accounting for the realistic non-Newtonian viscous behaviour of ceramic slurries is not available in the open literature. However, the simplified flow situation allows an analysis using Eringen's model of micropolar flow to be used, while still allowing a description of the effects arising from particle micromotions.

\section{Defining Equations}

The two-dimensional equations governing the isothermal, steady, laminar, incompressible micropolar fluid in a quiescent medium are given below. For details of the derivation see eg. Ramachandran et al. [8]. 


$$
\begin{gathered}
u \frac{\partial u}{\partial x}+v \frac{\partial u}{\partial y}=\left(\nu+\frac{\kappa}{\rho}\right)\left(\frac{\partial^{2} u}{\partial y^{2}}\right)+\frac{\kappa}{\rho} \frac{\partial N}{\partial y} \\
u \frac{\partial N}{\partial x}+v \frac{\partial N}{\partial y}=-\frac{\kappa}{\rho j}\left(2 N+\frac{\partial u}{\partial y}\right)+\frac{\nu_{s}}{\rho j} \frac{\partial^{2} N}{\partial y^{2}}
\end{gathered}
$$

In the above equations, $u$ and $v$ are the dimensional velocity components in the $x$ and $y$ directions, $N$ represents the microrotation whose direction of rotation is in the $x-y$ plane, $\nu$ and $\nu_{s}$ are respectively the viscosity and microrotation (or spin-gradient) viscosity, $j$ is the micro-inertia density, $\rho$ is the mass density of the fluid, $\kappa$ is the microrotation coupling coefficient (also known as the coefficient of gyroviscosity or as the vortex viscosity), and $p$ is the pressure. In the present work we assume that the microinertia density $j$ is constant.

To determine the conditions for which a similarity solution exists for this flow problem we introduce the transformations

$$
\begin{aligned}
\eta & =b y \\
\Psi & =a x F(\eta) \quad \Rightarrow \quad u=a b x F^{\prime} \quad \text { and } \quad v=-a F \\
N & =c x G(\eta)
\end{aligned}
$$

and we consider the general conditions $u(x, 0)=u_{w}$ and $v(x, 0)=v_{w}$ for the surface velocity components. However, in the present case the transformations invoked in $(3 \mathrm{~b})$ imply that the velocity normal to the sheet must be constant and the "stretching" velocity must vary linearly along the surface. 
For the microrotation, either the no-spin condition or the zero antisymmetric component of stress condition can be chosen as boundary conditions at the surface. In this work, the no-spin condition $N(x, 0)=0$ is employed. Outside the boundary layer, the appropriate boundary conditions are $\lim _{y \rightarrow \infty} v=0$ and $\lim _{y \rightarrow \infty} N=0$.

Equations (1) and (2) can now be simplified by choosing $a^{2}=\nu$ and $b^{2}=c^{2}=1 / \nu$, and by introducing the physical parameters $N_{1}=\kappa /(\rho \nu)$, $N_{2}=\nu_{s} /\left(\rho \nu^{2}\right)$ and $N_{3}=j / \nu$ along with the similarity transformations. We obtain

$$
\begin{aligned}
\left(F^{\prime}\right)^{2}-F F^{\prime \prime} & =\left(1+N_{1}\right) F^{\prime \prime \prime}+N_{1} G^{\prime} \\
N_{3}\left(F^{\prime} G-F G^{\prime}\right) & =N_{2} G^{\prime \prime}-N_{1}\left(F^{\prime \prime}+2 G\right)
\end{aligned}
$$

and the transformed boundary conditions are

$$
F^{\prime}(0)=1, \quad F(0)=-\frac{v_{w}}{a}=-V, \quad G(0)=0, \quad \lim _{\eta \rightarrow \infty} F^{\prime}=0, \quad \lim _{\eta \rightarrow \infty} G=0
$$

In the present work, the $N_{k}$ in (4) and (5) are treated as independent parameters.

For comparison with previous studies it proves convenient to rescale the functions and parameters using $F=\left(1+N_{1}\right) F_{1}, G=\left(1+N_{1}\right) F_{2}, C_{1}=$ 
$N_{1} /\left(1+N_{1}\right), C_{2}=N_{2} / N_{1}$ and $C_{3}=N_{3}$. Equations (4) and (5) are now

$$
\begin{aligned}
F_{1}^{\prime \prime \prime} & =-F_{1} F_{1}^{\prime \prime}+\left(F_{1}^{\prime}\right)^{2}-C_{1} F_{2}^{\prime} \\
C_{2} F_{2}^{\prime \prime} & =F_{1}^{\prime \prime}+2 F_{2}-\frac{C_{3}}{C_{1}}\left(F_{1} F_{2}^{\prime}-F_{2} F_{1}^{\prime}\right)
\end{aligned}
$$

In this work the values of $C_{1}, C_{2}$ and $C_{3}$ were chosen to be consistent with the inequalities $\mu, J>0$ and $\kappa, \nu_{s} \geq 0$ for micropolar fluids [3].

Specifically, the parameter $C_{3}$ is proportional to the square of a characteristic length of the microstructure. Consequently only very small values are of interest. For example, in the studies of Hassanien \& Gorla [5] and Hady [4] the term involving $C_{3}$ was neglected. Here, we also take $C_{3}=0$ and consider the simpler system

$$
\begin{aligned}
F_{1}^{\prime \prime \prime} & =-F_{1} F_{1}^{\prime \prime}+\left(F_{1}^{\prime}\right)^{2}-C_{1} F_{2}^{\prime} \\
C_{2} F_{2}^{\prime \prime} & =F_{1}^{\prime \prime}+2 F_{2}
\end{aligned}
$$

The parameter $C_{2}$, however, depends on the microrotational viscosity $\nu_{s}$ which could vary appreciably [6]. However, a limited range appears to have been considered in previous studies, with values near $C_{2}=2$ being most frequently chosen (see, eg. $[6,5,4]$ ).

Regarding our choice of $C_{2}$ values, preliminary results indicated that computations for $C_{2}>2$ were typically very stable and relatively easy to obtain. Therefore, we have mainly considered the computationally more demanding 
range $C_{2} \leq 2$ (numerical difficulties for small $C_{2}$ were also reported in [6]). In particular, values of $C_{2}=2,1 / 2,2 / 9$ and $1 / 8$ were chosen for comparison of numerical results with the perturbation analysis described below.

Finally, we note that $C_{1}$ can take the range of values $0 \leq C_{1}<1$, where $C_{1}=0$ corresponds to the uncoupled case $\kappa=N_{1}=0$ (where the macroscopic motion is unaffected by the microrotations) and $C_{1}=1$ corresponds to the limiting case of an infinite coupling parameter (ie. $\kappa, N_{1} \rightarrow \infty$ ). This allowable range of values of $C_{1}$ motivates the perturbation analysis presented in the next section.

\section{Perturbation Analysis}

Choosing $C_{1}$ as the perturbing parameter, we expand the similarity functions using the regular perturbation expansions

$$
\begin{aligned}
& F_{1}=f_{0}+C_{1} f_{1}+C_{1}^{2} f_{2}+\cdots \\
& F_{2}=g_{0}+C_{1} g_{1}+C_{1}^{2} g_{2}+\cdots
\end{aligned}
$$

and substitute them into the boundary-layer equations (8).

By collecting terms in equal powers of $C_{1}$, a hierarchy of ordinary differential equations for the functions $f_{n}$ and $g_{n}$ can be obtained. The first four 
equations for $f_{n}$ are as follows.

$$
\begin{aligned}
& f_{0}^{\prime \prime \prime}+f_{0} f_{0}^{\prime \prime}-\left(f_{0}^{\prime}\right)^{2}= 0 \\
& f_{n}^{\prime \prime \prime}+f_{0} f_{n}^{\prime \prime}-2 f_{0}^{\prime} f_{n}^{\prime}+f_{0}^{\prime \prime} f_{n}= \begin{cases}-g_{0}^{\prime} & \text { if } n=1 \\
-g_{1}^{\prime}-f_{1} f_{1}^{\prime \prime}+\left(f_{1}^{\prime}\right)^{2} & \text { if } n=2, \\
-g_{2}^{\prime}-f_{1} f_{2}^{\prime \prime}-f_{2} f_{1}^{\prime \prime}+2 f_{1}^{\prime} f_{2}^{\prime} & \text { if } n=3\end{cases}
\end{aligned}
$$

For $n \geq 0$, the microrotation functions $g_{n}$ may be obtained via solution of the following second order equation.

$$
C_{2} g_{n}^{\prime \prime}-2 g_{n}=f_{n}^{\prime \prime}
$$

The appropriate boundary conditions for equations (10) and (11) are

$$
\begin{array}{lrlrl}
f_{0}(0)=-V_{1}, & f_{0}^{\prime}(0) & =1, \quad g_{0}(0)=0, & & \\
\lim _{\eta \rightarrow \infty} f_{0}^{\prime}=0, & \lim _{\eta \rightarrow \infty} g_{0} & =0, & & \text { for } n=0, \\
f_{n}(0)=0, & f_{n}^{\prime}(0) & =0, & g_{n}(0)=0, & \\
\lim _{\eta \rightarrow \infty} f_{n}^{\prime}=0, & \lim _{\eta \rightarrow \infty} g_{n}=0, & & \text { for } n>0 .
\end{array}
$$

\subsection{Zeroth Order Solution $(n=0)$ for $F_{1}$ and $F_{2}$}

The leading order equation for the stream function given in (10a) is a nonlinear, third order and autonomous ordinary differential equation. A closed 
form solution for $f_{0}$ which satisfies the relevant boundary conditions is $[7]$

$$
f_{0}=-V_{1}+\frac{1-\exp (-\beta \eta)}{\beta}=\beta(1-\omega)
$$

where $\beta=\frac{1}{2}\left(-V_{1}+\sqrt{V_{1}^{2}+4}\right)$ and $\omega=\omega(\eta)=\frac{\exp (-\beta \eta)}{\beta^{2}}$

To obtain the equation for the zeroth order micro-rotation $g_{0}$, we let $r^{2}=2 / C_{2}$ and introduce (13) into equation (11) with $n=0$ to obtain

$$
g_{0}^{\prime \prime}-r^{2} g_{0}=-\frac{\beta^{3} r^{2}}{2} \omega
$$

For the solution of this linear, second order and non-homogeneous ordinary differential equation two cases must be considered, depending on the relative values of the two positive parameters $r$ and $\beta$. Introducing the parameter $\gamma=r / \beta$, solution of (14) yields

$$
\begin{aligned}
& g_{0}=\frac{\beta^{4}}{4} \eta \omega \\
& g_{0}^{\prime}=\frac{\beta^{4}}{4}(1-\beta \eta) \omega
\end{aligned}
$$


when $\gamma=1$, and

$$
\begin{aligned}
& g_{0}=\frac{\beta^{3}}{2} \frac{\gamma^{2}}{\gamma^{2}-1}\left(\omega-\beta^{2 \gamma-2} \omega^{\gamma}\right) \\
& g_{0}^{\prime}=-\frac{\beta^{4}}{2} \frac{\gamma^{2}}{\gamma^{2}-1}\left(\omega-\gamma \beta^{2 \gamma-2} \omega^{\gamma}\right)
\end{aligned}
$$

in the more general case where $\gamma \neq 1$. It is straight forward to show that in the limit as $\gamma \rightarrow 1$, the expressions for $g_{0}$ and $g_{0}^{\prime}$ given in (16) simplify to those given in (15), as expected.

\subsection{First Order Solution $(n=1)$ for $F_{1}$}

For $n>0$, the differential equation (10b) for $f_{n}$ is non-homogeneous, third order and linear with variable coefficients. For the case $n=1$, a closed form general solution can be obtained, and can be expressed as $f_{1}=h_{c}+p_{1}$, where $h_{c}$ is the complementary function $(\mathrm{CF})$ and $p_{1}$ is a particular integral (PI). These are discussed below.

\subsubsection{Complementary Function}

For all $n>0$, the $\mathrm{CF}$ is obtained via the solution of the homogeneous part of $(10 \mathrm{~b})$, ie.

$$
h_{c}^{\prime \prime \prime}+f_{0} h_{c}^{\prime \prime}-2 f_{0}^{\prime} h_{c}^{\prime}+f_{0}^{\prime \prime} h_{c}=0
$$


and can be expressed as a linear combination of three independent solutions as $h_{c}=a_{1} h_{c 1}+a_{2} h_{c 2}+a_{3} h_{c 3}$, where $a_{1}, a_{2}$ and $a_{3}$ are constants.

Two of the solutions which comprise the CF are in simple closed form as

$$
\begin{array}{ll}
h_{c 1}=\omega, & h_{c 1}^{\prime}=-\beta \omega \\
h_{c 2}=1+\beta \eta \omega, & h_{c 2}^{\prime}=\beta \omega(1-\beta \eta)
\end{array}
$$

while the third independent solution can be expressed in terms of quadratures as

$$
\begin{aligned}
& h_{c 3}=-2 \exp (-\omega)+\operatorname{Ei}(\omega)+2 \omega \operatorname{Ei}(\omega)+\omega \int_{\omega} \frac{\operatorname{Ei}(t)}{t} d t \\
& h_{c 3}^{\prime}=\beta \exp (-\omega)-\beta \omega \operatorname{Ei}(\omega)-\beta \omega \int_{\omega} \frac{\operatorname{Ei}(t)}{t} d t
\end{aligned}
$$

where $\operatorname{Ei}(x)$ is the exponential integral function. The derivation of the above results is somewhat lengthy, and further details can be found in [7].

In order to introduce algebraic simplifications, and without loss of generality, we use here a modified definition of

$$
\operatorname{Ei}(x)=\int_{x}^{1 / \beta^{2}} \frac{e^{-t}}{t} d t
$$

in $(18 \mathrm{c})$, where the usual upper limit of positive infinity has been replaced by $1 / \beta^{2}$. We also set the upper limit to $1 / \beta^{2}$ for any integral of the exponential integral that may arise, such as occurs in equation (18c). 


\subsubsection{Particular integral}

We consider now a particular integral to equation (10b) when $n=1$. For this third order, non-homogeneous ode two cases of $\gamma=1$ and $\gamma \neq 1$ must again be considered.

For $\gamma=1$, the expression for $g_{0}^{\prime}$ given in (15) is substituted into the RHS of (10b) and a PI $p_{1}$ is sought. The latter can be found by considering a trial solution of the form $p_{1}=c_{0}+c_{1} \eta$, which on substitution into (10b) yields the solution given in Table 1.

Having obtained a CF and PI for this case, a general solution which satisfies the necessary boundary conditions can be obtained by solving for the constants $a_{1}, a_{2}$ and $a_{3}$. The required values can be found with the assistance of a CAS, and are given in Table 2 . Note that in the table, and subsequently, we have used $a_{0}=1-\exp \left(1 / \beta^{2}\right)$ and $b_{0}=\log \left(1 / \beta^{2}\right)$.

For the more general case where $\gamma \neq 1$, the PI is of considerable complexity, and was found with the aid of a CAS (we used Mathematica 3) using a mixture of online and offline analysis. After completion of the analysis, the PI for $\gamma \neq 1$ was found in terms of nested quadratures. At the expense of a loss of generality, the PI can be found in terms of no quadratures if integer values of $\gamma$ are taken. The resulting expressions are easy to evaluate, and the solutions for $p_{1}$ for $\gamma=2,3,4$ are given in Table 1 . For $\beta=1$ (an impermeable sheet), these choices correspond to the values $C_{2}=1 / 2,2 / 9$ and $1 / 8$ indicated earlier. The two smaller of these values, in particular, extend upon 
TABLE 1: Particular integral for $n=1$ and $\gamma=1 \rightarrow 4$.

\begin{tabular}{|c|c|}
\hline$\gamma$ & Particular integral $p_{1}$ \\
\hline 1 & $p_{1}=\frac{3 \beta}{4}-\frac{\beta^{2}}{4} \eta$ \\
\hline 2 & $\begin{aligned} p_{1}= & \frac{2 \beta}{3}\left(-4 \beta^{2}-\omega \log (\omega)-2 \beta^{2} \log (\omega)-4 \beta^{2} \omega \log (\omega)\right. \\
& \left.+\beta^{2} \omega[\log (\omega)]^{2}\right)\end{aligned}$ \\
\hline 3 & $\begin{aligned} p_{1}= & \frac{9 \beta}{16}\left(24 \beta^{4}+3 \beta^{4} \omega^{2}+12 \beta^{4} \log (\omega)-\left(1-24 \beta^{4}\right) \omega \log (\omega)\right. \\
& \left.-6 \beta^{4} \omega[\log (\omega)]^{2}\right)\end{aligned}$ \\
\hline 4 & $\begin{aligned} p_{1}= & \frac{8 \beta}{15}\left(-144 \beta^{6}-18 \beta^{6} \omega^{2}+\beta^{6} \omega^{3}-72 \beta^{6} \log (\omega)\right. \\
& \left.-\left(1+144 \beta^{6}\right) \omega \log (\omega)+36 \beta^{6} \omega[\log (\omega)]^{2}\right)\end{aligned}$ \\
\hline
\end{tabular}

the range of $C_{2}$ values used in previous studies.

Finally, once the $\mathrm{CF}$ and PI are known, the values of the constants in the general solution may be determined. Here again, the labour involved is significantly reduced by using a CAS, and the required values are given in Table 2.

The values of the constants given in Table 2, along with equations (18a, $18 \mathrm{~b}, 18 \mathrm{c}$ ) and the particular integrals given in Table 1, completely specify the solution of the first order streamfunction $f_{1}$ for the values of $\gamma$ considered here. 
TABLE 2: Values for the constants $a_{i}$ for $n=1$ and $\gamma=1 \rightarrow 4$.

\begin{tabular}{|l|l|l|}
\hline$\gamma=1$ & $a_{1}$ & $-\frac{\beta^{3}}{4}\left(a_{0}+\frac{1+a_{0}}{1+\beta^{2}}\right)$ \\
& $a_{2}$ & $-\frac{\beta^{3}}{4}\left(\frac{1+a_{0}}{1+\beta^{2}}\right)$ \\
& $a_{3}$ & $\frac{\beta}{4}$ \\
\hline$\gamma=2$ & $a_{1}$ & $\frac{2 \beta}{3}\left(-3+4 \beta^{2}+2 a_{0} \beta^{4}+b_{0}+4 \beta^{2} b_{0}-\beta^{2} b_{0}^{2}+\frac{3+2 a_{0} \beta^{4}}{1+\beta^{2}}\right)$ \\
& $a_{2}$ & $\frac{2 \beta}{3}\left(-4+2 \beta^{2} b_{0}+\frac{3+2 a_{0} \beta^{4}}{1+\beta^{2}}\right)$ \\
& $a_{3}$ & $-\frac{4 \beta^{3}}{3}$ \\
\hline$\gamma=3$ & $a_{1}$ & $\frac{9 \beta}{16}\left(-20+18 \beta^{2}-24 \beta^{4}-12 a_{0} \beta^{6}+b_{0}\right.$ \\
& & $\left.-24 \beta^{4} b_{0}+6 \beta^{4} b_{0}^{2}+\frac{20-12 a_{0} \beta^{6}}{1+\beta^{2}}\right)$ \\
& $a_{2}$ & $\frac{9 \beta}{16}\left(-21+24 \beta^{2}-12 \beta^{4} b_{0}+\frac{20-12 a_{0} \beta^{6}}{1+\beta^{2}}\right)$ \\
\hline$\gamma=4$ & $a_{3}$ & $\frac{27 \beta^{5}}{4}$ \\
\hline & $a_{1}$ & $\frac{8 \beta}{15}\left(-123+123 \beta^{2}-108 \beta^{4}+144 \beta^{6}+72 a_{0} \beta^{8}\right.$ \\
& & $\left.+b_{0}+144 \beta^{6} b_{0}-36 \beta^{6} b_{0}^{2}+\frac{123+72 a_{0} \beta^{8}}{1+\beta^{2}}\right)$ \\
& $a_{2}$ & $\frac{8 \beta}{15}\left(-124+126 \beta^{2}-144 \beta^{4}+72 \beta^{6} b_{0}+\frac{123+72 a_{0} \beta^{8}}{1+\beta^{2}}\right)$ \\
& $a_{3}$ & $-\frac{192 \beta^{7}}{5}$ \\
\hline
\end{tabular}




\section{Numerical method}

As a cross-check for the analysis, a shooting method using a fourth-order Runge-Kutta algorithm and a quasilinearisation scheme were used here. For both methods, discretisation errors were reduced by selecting step sizes and convergence criteria small enough so that results accurate to more than 5 significant digits could be reported.

For brevity, an outline of the quasilinearisation method only is now given. Further details can be found in [2].

Let $f_{(j)}, g_{(j)}$ be approximate solutions and $f_{(j+1)}, g_{(j+1)}$ improved solutions for $F_{1}$ and $F_{2}$ respectively in equation (8). After linearization, the following two coupled linear equations are obtained.

$$
\begin{aligned}
f_{(j+1)}^{\prime \prime \prime}= & {\left[-f_{(j+1)}^{\prime \prime} f_{(j)}+2 f_{(j+1)}^{\prime} f_{(j)}^{\prime}-f_{(j+1)} f_{(j)}^{\prime \prime}-C_{1} g_{(j+1)}^{\prime}\right] } \\
& +\left(f_{(j)}^{\prime \prime} f_{(j)}-\left(f_{(j)}^{\prime}\right)^{2}\right) \\
C_{2} g_{(j+1)}^{\prime \prime}= & {\left[2 g_{(j+1)}+f_{(j+1)}^{\prime \prime}\right] }
\end{aligned}
$$

The numerical solution is obtained using the linear combination $S=$ $S_{p}+k_{1} S_{h 1}+k_{2} S_{h 2}$ where $S_{p}$ and the $S_{h i}$ are respectively the particular integral and complementary functions of (20). The complementary function consists of two linearly independent functions $S_{h 1}$ and $S_{h 2}$ which are solutions of the homogeneous part of (20). 
At each stage of the iterative procedure, the required conditions for $S_{p}$ and $S_{h i}$ at the surface are

$$
\begin{aligned}
\left.S_{p}(0)_{(j+1}\right) & =\left[-V, 1, f_{(j)}^{\prime \prime}(0), 0, g_{(j)}^{\prime}(0)\right]^{T} \\
S_{h 1}(0) & =[0,0,1,0,0]^{T} \\
S_{h 2}(0) & =[0,0,0,0,1]^{T}
\end{aligned}
$$

To satisfy the conditions at infinity (implemented on a finite domain of length $\eta_{c}$ ), weighting coefficients $k_{1}$ and $k_{2}$ are calculated at each stage at the cutoff point $\eta=\eta_{c}$ from

$$
\begin{aligned}
& 0=f_{p}^{\prime}+k_{1} f_{h 1}^{\prime}+k_{2} f_{h 2}^{\prime} \\
& 0=g_{p}+k_{1} g_{h 1}+k_{2} g_{h 2}
\end{aligned}
$$

After a solution to (20) subject to (21) and (22) has been obtained it is compared with the solution at the previous step. If convergence has not been achieved or greater accuracy is required, the procedure above is repeated.

To initiate the iterative scheme, an initial approximate solution is needed. For the majority of results obtained here, we have used the solution given in equation (13) for this purpose. In general, however, any weak solution to the governing equations may be used. 


\section{Results}

As part of this study, the accuracy, convergence and other computational properties of the numerical schemes used here were thoroughly investigated. For brevity, only our key findings are described here. In addition, the results given below are confined to the case $\beta=1$, ie. an impermeable stretching sheet.

\subsection{Results for $C_{1}=0$}

In Figure 1 the influence of the parameter $C_{2}$ is shown when $C_{1}=0$. For all values of $C_{2}$ considered here, the computed results for $F_{1}$ shown in Figure 1(a) using either Runge-Kutta or Quasilinearisation schemes were in excellent agreement with the leading order solution given in (13).

In general, a computed solution for $F_{1}$ which matches (13) was usually obtained if an initial guess of $-F_{1}^{\prime \prime}(0) \approx \beta$ was used. However, in some cases an apparently spurious numerical solution for $F_{1}$ which differs from (13) was computed, most frequently if the iterative schemes were initiated with $-F_{1}^{\prime \prime}(0)$ slightly larger than $\beta$. This alternative solution for $F_{1}$ is also shown in Figure 1(a), as computed on domain lengths ranging from $\eta_{c}=25$ to 125 . It is unclear whether this second solution represents an asymptotically valid solution to the governing equations. Although the observed changes are small as $\eta_{c}$ is increased, it is possible that the second solution will eventually 


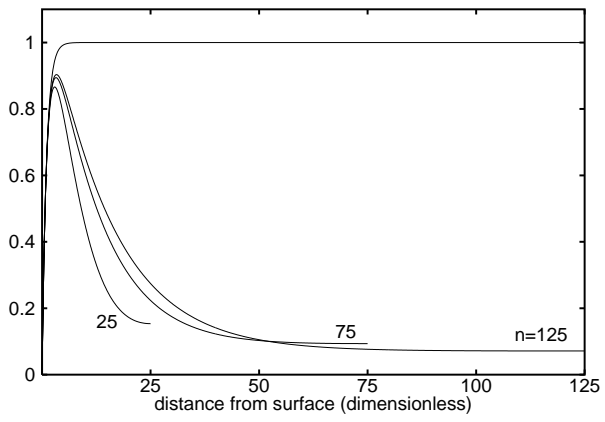

(a) $F_{1}\left(\eta_{c}=25 \rightarrow 125\right)$

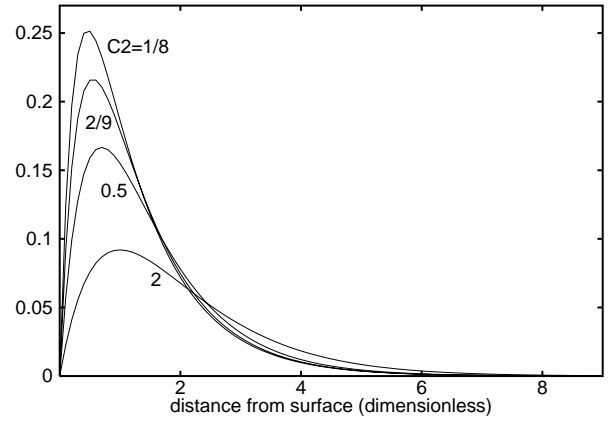

(b) $F_{2}\left(C_{2}=2,1 / 2,2 / 9,1 / 8\right)$

Figure 1: Profiles of Streamfunction $F_{1}$ and Microrotation $F_{2}$ for $C_{1}=0$ and various $C_{2}$.

converge to the envelope curve described by (13) as $\eta_{c} \rightarrow \infty$. Although not shown here, for the case where $C_{1} \neq 0$ we also found apparently spurious numerical solutions where the solution for $F_{1}$ has a similar profile to those shown in Figure 1(a).

In Figure 1(b) the influence of the parameter $C_{2}$ on the computed solution for the microrotation $F_{2}$ is shown. Here again, the computed results for $F_{2}$ shown were found to be in excellent agreement with the leading order solution given in (15) and (16).

From Figure 1(b), the observed behaviour is that the micro-rotation profile both flattens and broadens as $C_{2}$ increases. Although not shown, this 
trend was evident in results for $C_{2}=5,10$ and 50 as well, and is consistent with a leading order analysis that $F_{2} \approx 0$ for $C_{2}$ large, presented in [7].

For $C_{2}>2$ the computations proved to be very stable, and converged without difficulty to a final solution even if very poor initial conditions were used to initiate the iterative schemes. However, for $C_{2}<2$ the opposite was true. Accurate initial conditions were required. Further, as $C_{2}$ was decreased, we also found that we needed to reduce the domain length $\eta_{c}$ to avoid instability. The cutoff errors introduced by using shorter domains were noticeable for $C_{2}=1 / 8$, where the computed solution agreed with the leading order solution for $F_{2}$ given in (16) to an accuracy of about 3 significant digits only.

As $C_{2}$ was decreased, we observed that the primary source of numerical instability arose from the computation of the microrotation equation. The observed increase in numerical difficulties seems consistent with the boundary layer behaviour of the profiles shown in Figure 1(b). Heruska et al. [6] also noted computational difficulties for small $C_{2}$ using a globally convergent homotopy method, but did not suggest any reasons for this behaviour.

\subsection{Results for $C_{1} \neq 0$}

We now consider the influence of the parameter $C_{2}$ for values of $C_{1}$ other than zero. For brevity, sample results for $C_{1}=0.1$ only are presented below in order to establish the main findings (for other results see [7]). 


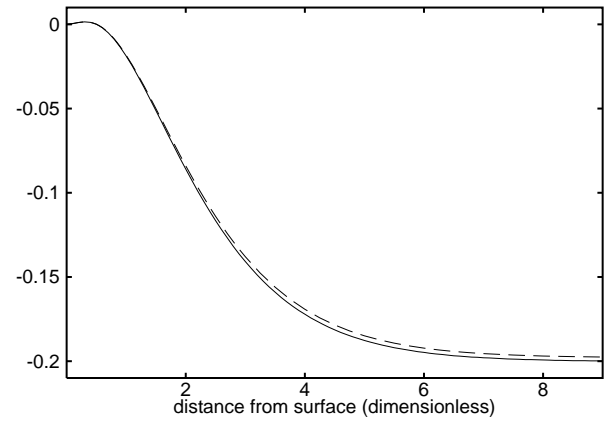

(a) $\left(F_{1}-f_{0}\right) / C_{1}$ and $f_{1}\left(C_{2}=2 / 9\right)$

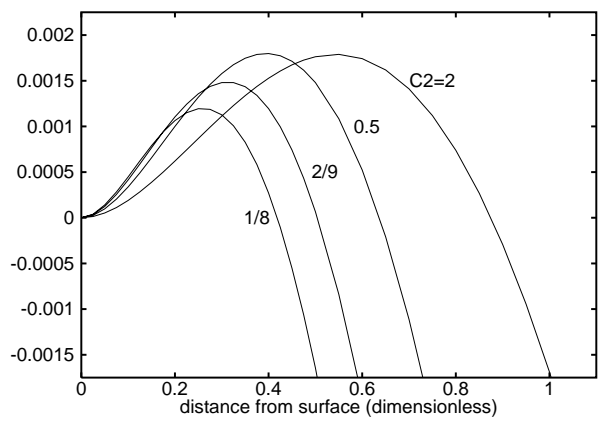

(b) $f_{1}$ for various $C_{2}$

Figure 2: (a) Comparison of profiles of $\left(F_{1}-f_{0}\right) / C_{1}$ (continuous) and $f_{1}$ (dashed) for $C_{1}=0.1$ and $C_{2}=2 / 9$; (b) detail of $f_{1}$ profiles for $C_{2}=$ $2,1 / 2,2 / 9$ and $1 / 8$. 
In Figure 2(a) the computed profiles for $F_{1}$ and $C_{2}=2 / 9$ are compared with the corresponding perturbation solution. For clarity, results for $F_{1}$ using $\left(F_{1}-f_{0}\right) / C_{1}$ are compared with $f_{1}$. For this case, and for $C_{2}=2,1 / 2$ and $1 / 8$ (not shown), the differences between the compared profiles are very small. It is clear that for $C_{1}=0.1$, the solution for $F_{1}$ using a first order perturbation series $f_{0}+C_{1} f_{1}$ yields a very good approximation to the computed solution of the governing equations.

With regard to stability, computations for the case $C_{1}=0.1$ behaved in a similar way to those for $C_{1}=0$. We again had to successively reduce the domain of integration to avoid non-convergence, particularly for the cases $C_{2}=2 / 9$ and $1 / 8$. Compared with the results for $C_{1}=0$, correspondingly shorter domains were needed, indicating that the computations were relatively less stable for $C_{1}=0.1$.

In Figure 2(b) we show the complex behaviour of the perturbation solutions for $f_{1}$ near the surface. In contrast to the computational approaches used here, the data for these solution curves can be obtained using a CAS without any numerical difficulties. It is clear that very close to the surface there is initially a small positive contribution from $f_{1}$ to the series solution for $F_{1}$ given in (9). Physically, the profiles indicate that when the coupling constant $C_{1} \neq 0$, the microrotational effects lead to a reduction in the skin friction. The latter is observed in eg. fluids with extremely small polymeric additives [3].

We also observe that as $C_{2}$ decreases, the region where $f_{1}>0$ also de- 
creases in transverse extent. Although not entirely clear from the figure, the value of $f_{1}^{\prime \prime}(0)$ (which to first order is directly proportional to the reduction in skin friction) actually increases as $C_{2}$ decreases.

Finally, we note that the near wall behaviour of these profiles as $C_{2}$ decreases is similar to that of a boundary layer where the boundary layer width is decreasing. Compared with the uncoupled case $C_{1}=0$, it is possible that the observed increase in numerical difficulties for $C_{1}=0.1$ is due to this more complex behaviour.

\section{Conclusion}

In this paper, we have investigated the solutions to the boundary-layer equations for the micropolar fluid flow over a stretching sheet. We have considered in some detail the influence of the physical parameters on the similarity solutions via a perturbation analysis and a numerical approach using RungeKutta and quasilinearisation schemes.

We found that decreasing the value of the parameter $C_{2}$ leads to an intensification of the microrotation in an increasingly narrow region adjacent to the surface. For the case of a non-zero coupling parameter $C_{1}$, a more complex near-wall profile for the streamfunction $F_{1}$ is also obtained. For this case, reducing the value of $C_{2}$ leads to successive reductions in the skin friction at the surface for the range considered. 
For the smaller of the $C_{2}$ values considered here, and also for the case where $C_{1} \neq 0$, the presence of some instability in the numerical schemes used here was attributed to the more complex near-wall flow behaviour. In contrast, the perturbation analysis gave analytic solutions which, although rather involved, could be evaluated without difficulty to provide data about this complex flow, especially for parameter values where a numerical approach proved computationally more difficult.

\section{References}

[1] T. Ariman, M. A. Turk, and N. D. Sylvester. Microcontinuum fluid mechanics - a review. Int. J. Engng Sci., 11:905-930, 1973. C538

[2] A. Desseaux. Numerical contribution to a problem of convection in porous media with lateral mass flux. In Proc. Ninth Int. Symp. on Transport Penomena in Thermal-Fluids Eng., pages 994-999. Singapore, 1996. C551

[3] A. C. Eringen. Theory of micropolar fluids. J. Mathematics 86 Mechanics, 16:1-18, 1966. C538, C542, C557

[4] F. M. Hady. On the solution of heat transfer to micropolar fluid from a non-isothermal stretching sheet with injection. Int. J. Num. Meth. Heat Fluid Flow, 6:99-104, 1996. C542, C542 
[5] I. A. Hassanien and R. S. R. Gorla. Heat transfer to a micropolar fluid from a non-isothermal stretching sheet with suction and blowing. Acta Mechanica, 84:191-199, 1990. C542, C542

[6] M. W. Heruska, L. T. Watson, and K. K. Sankara. Micropolar flow past a porous stretching sheet. Computers \& Fluids, 14:117-129, 1986. C542, C542, C543, C555

[7] N. A. Kelson, A. Desseaux, and D. L. S. McElwain. Limiting solutions and linearised analysis of micropolar fluid flow driven by a porous stretching surface. Submitted. C545, C547, C555, C555

[8] P. S. Ramachandran, M. N. Mathur, and S. K. Ojha. Heat transfer in boundary layer flow of a micropolar fluid past a curved surface with suction and injection. Int. J. Engng. Sci., 17:625-639, 1979. C539 